\title{
Magnetic quenching of positronium in thorium phosphate: an experimental approach to p-Ps pick-off lifetime?
}

\author{
T. GOWOREK ${ }^{(1)}$, A. BADIA and G. DUPLÂTRE
}

Laboratoire de Chimie Nucléaire, C.R.N., IN2P3 and Université Louis Pasteur, BP. 20, 67037 Strasbourg, France

\begin{abstract}
The effects of magnetic field (B) quenching on positronium (Ps) are studied in solid thorium phosphate by using both the lifetime spectroscopy (LS) and Doppler broadening (DBARL) techniques, in view of gaining information on the pick-off decay rate constant of positronium singlet state (p-Ps) and compare it to that of the triplet state (o-Ps). At $B=0$, the longest lifetime is $\tau_{3}^{\circ} \approx 700$ ps, with an intensity of some $23 \%$. The effects of B on LS parameter R (normalized integral of counts in a time window) show that $\tau_{3}^{\circ}$ is ascribable to o-Ps. Fitting the variations with $B$ of both $R$ and $\tau_{4}$ (lifetime of the $m=0$ o-Ps substate) requires two phenomenological parameters : (i) the contact density parameter, $\eta \approx 0.22$ and (ii) either an additional contact density parameter, $\eta^{\prime} \approx 0.36$, or

a p-Ps pick-off decay constant, $\lambda_{\text {pol }} \approx 2.59 \mathrm{~ns}^{-1}$, different from that of o-Ps, $\lambda_{\text {po } 3}=1 / \tau_{3}^{\circ}$. Resolving the deconvoluted DBARL spectra to derive the intensity of the intrinsic decay component at $\mathrm{B}=0$ gives a better agreement with the first $\left(\eta, \eta^{\prime}\right)$ than with the second $(\eta, \lambda$ pol $)$ of these possibilities.
\end{abstract}

1.Introduction. Although essentially intrinsic, the annihilation of p-Ps in matter involves some contribution from pick-off, the dominant annihilation mode of o-Ps by which the positron in positronium annihilates with a strange electron of the atoms of the surrounding medium. Whether the p-Ps and o-Ps pick-off decay rate constants are equal or not is still controversial. Among other aspects, this question is relevant to the o-Ps/p-Ps formation ratio as inferred from measurements of the o-Ps (through LS) and p-Ps (through angular correlations, AC, or through DBARL) intensities : those p-Ps atoms which decay via pick-off annihilation do not participate to the narrow component of the momentum spectra.

On passing from vacuum to matter, any change in the overlap between the positron and electron wave functions in Ps may be expressed through the phenomenological contact density parameter, $\eta$ $(\eta=1$ in vacuo). Theory predicts that $\eta$ should affect both the Ps hyperfine splitting and intrinsic lifetime. From experiments on magnetic field effects, it is known that in solids $\eta$ is often rather low (Ps is swollen) : for p-Ps, this results in a high pick-off/intrinsic decay rate ratio as compared to liquids, where usually $\eta$ is close to 1 . It thus appeared promising to gain information on the p-Ps pick-off rate constant by examining the effects of B on Ps in a solid displaying a short o-Ps pick-off lifetime, using both LS and DBARL.

2. Experimental. The solid matrix was polycrystalline thorium phosphate prepared at high temperature and compressed into pellets. The LS spectrometer (plastic scintillators with light guides) had a resolution of 380 ps (1). At $B=0$, complementary measurements were made using 
spectrometers with resolutions of 310 ps (plastic) or $215 \mathrm{ps}\left(\mathrm{BaF}_{2}\right)$. At $\mathrm{B}=0$ (denoted by superscript "o"), the LS spectra include 3 components of intensities $I_{i}^{\circ}$ and lifetimes $\tau_{i}^{\circ}$ (decay constants : $\lambda_{i}^{\circ}=$ $\left.1 / \tau_{i}^{\circ}\right)$; subscripts $i=1,2$ and 3 refer to $p-P s$, free $\mathrm{e}^{+}$and o-Ps, respectively. At $B \neq 0$, the mixing of the $m=0$ substates of Ps results in a decrease of the o-Ps $(m=0)$ lifetime $\left(\tau_{4} ; I_{4}=I_{3}^{\circ} / 3\right)(2)$ :

$$
\lambda_{4}=1 / \tau_{4}=\left(\mathrm{y}^{2} \lambda_{1}^{\circ}+\lambda_{\mathrm{po} 3}\right) /\left(1+\mathrm{y}^{2}\right)
$$

with : $\quad \lambda_{1}^{0}=\eta^{\prime} \lambda_{\mathrm{s}}+\lambda_{\mathrm{po} 1}$

and : $\quad y=\left[\left(1+x^{2}\right)^{1 / 2}-1\right] / x, \quad x=0.02756 \mathrm{~B}(\mathrm{~T}) / \eta$

In the absence of any excited Ps states, one has $\eta=\eta^{\prime}$; the pick-off decay constants are denoted by $\lambda_{\text {po } 3}=\lambda_{3}^{\circ}$ for o-Ps and $\lambda_{\text {pol } 1}$ for p-Ps, while $\lambda_{\mathrm{s}}=8 \mathrm{~ns}^{-1}$ is the intrinsic p-Ps decay constant.

The LS data at $\mathrm{B} \neq 0$ are expressed either through $\tau_{4}$, obtained by fixing the parameters for o-Ps $(m= \pm 1)\left(\tau_{3}, I_{3}=2 I_{3}^{\circ} / 3\right)$ and $I_{4}$, or through parameter $R$, which is the normalized ratio of integral of counts in a time window $\left(t_{\mathrm{a}}, \mathrm{t}_{\mathrm{b}}\right)$ chosen such as essentially only the longest lived components are present. Experimental error on $\tau_{4}: 40 \mathrm{ps}$; on $\mathrm{R}: 0.013$.

The DBARL data were collected using an hyperpure Ge detector with a resolution of $1.38 \mathrm{keV}$ at $514 \mathrm{keV}\left({ }^{85} \mathrm{Sr}\right.$ line). The spectra at $\mathrm{B}=0$ were deconvoluted and resolved into 3 gaussian components with intensities $I_{i}^{D}$ and fwhm's $\Gamma_{\mathrm{i}}$ (a weak broad component was also present, as is usual in liquids too). Knowing $I_{2}^{\mathrm{D}}=\left(100-4 I_{3}^{\circ} / 3\right) \%$ from LS allows to derive $\Gamma_{2}$, while $\Gamma_{1}$ and $\Gamma_{3}$ can be obtained with $\mathrm{I}_{1}^{\mathrm{D}}$ and $\mathrm{I}_{3}^{\mathrm{D}}$ either free or fixed to values calculated on working hypotheses.

\section{Results and discussion.}

3.1. LS data. Even at $B=0, \tau_{3}^{\circ}$ is rather short (table 1), indicating that $P$ is trapped in a small void in the thorium phosphate lattice, with a radius of about $0.11 \mathrm{~nm}$. Because of the magnetic quenching, both $\mathrm{R}$ (fig. 1 ) and $\tau_{4}$ decrease with increasing $B$.

Although acceptable in view of the $\sigma$ values (see table 1 ), the hypothesis of a single parameter (with $\eta=\eta^{\prime}$, treatment $c$ ) does not give good results for the fitting of $R$ and $\tau_{4}$; in particular, the calculated variation of $R$ is systematically too low at low $B$, and too high at $B>1 T$.

Table 1. LS parameters at $B=0$ and parameters derived from the effects of $B$ on $R$ and $\tau_{4}\left(\eta, \eta^{\prime}\right.$, $\left.\tau_{\text {pol }}\right)$ together with the resulting standard deviations $(\sigma) ; \mathrm{t}_{\mathrm{a}}=1.6 \mathrm{~ns}, \mathrm{t}_{\mathrm{b}}=5 \mathrm{~ns}$.

\begin{tabular}{lllllll}
\hline$\tau_{2}^{\circ}(\mathrm{ps})$ & (a) $336 \pm 15$ & (b) $322 \pm 10$ & (c) $\leq 319$ & (d) fixed to 322 \\
$\tau_{3}^{\circ}(\mathrm{ps})$ & (a) $735 \pm 33$ & (b) $696 \pm 17$ & (c) $646 \pm 35$ & (d) $689 \pm 20$ \\
$\mathrm{I}_{3}^{\circ}(\%)$ & (a) $20.1 \pm 1.6$ & (b) $23.6 \pm 1.2$ & (c) $23 \pm 3$ & (d) & $22.7 \pm 1.3$ \\
$\eta$ & (c) $0.32 \pm 0.02\left(\sigma_{\tau 4}=40 \mathrm{ps} ; \sigma_{\mathrm{R}}=0.021\right)$ & & & \\
& (d) $0.22 \pm 0.06\left(\sigma_{\tau 4}=17 \mathrm{ps} ; \sigma_{\mathrm{R}}=0.010\right)$ & & & \\
$\eta^{\prime}$ & (h1) 0.36 & $\tau_{\mathrm{pol}}(\mathrm{ps})$ & (h2) 386 & & \\
\hline
\end{tabular}

(a) all results (15 measurements); (b) $\mathrm{BaF}_{2}$ spectrometer ; (c) from fitting of $\mathrm{R}$ and $\tau_{4}$ with $\eta=\eta^{\prime}$ and $\tau_{\mathrm{po} 1}=\tau_{\mathrm{po} 3} ;$ (d) from fitting of $R$ and $\tau_{4}$ with $\eta \neq \eta^{\prime}(\mathrm{h} 1)$ or $\tau_{\mathrm{pol} 1} \neq \tau_{\mathrm{po} 3}(\mathrm{~h} 2)$, see eq.(2). 
From eq.(2), two working hypotheses (h1, h2), both defining $\lambda_{i}^{\circ}$, are possible to fit the data with two parameters ; either $\eta \neq \eta^{\prime}$ and $\tau_{\text {po } 3}=\tau_{\text {pol }}(\mathrm{h} 1)$, or $\eta=\eta^{\prime}$ and $\tau_{\text {po } 3} \neq \tau_{\text {pol }}$ (h2). In this case (h1 or h2), there is an excellent agreement for the variations of $R$ (solid line in fig. 1) and $\tau_{4}$ with $B$. The results are similar whether $R$ and $\tau_{4}$ are fitted alone or together, and with $t_{a}=1.6$ or $1.8 \mathrm{~ns}$.

3.2. DBARL results. Only the hypothesis of two parameters was considered. By contrast with the LS data, the DBARL results should allow to discriminate between having either $\eta$ and $\eta^{\prime}(\mathrm{hl}$ ) or $\eta$ and $\tau_{\text {po1 }}$ (h2) as fitting parameters, through the theoretical expression of $I_{1}^{D}(3)$; thus, at $B=0$ :

for h1 $\quad I_{1}^{D}=\left(I_{3}^{\circ} / 3\right) \eta^{\prime} \lambda_{s} /\left(\eta^{\prime} \lambda_{s}+\lambda_{\text {po3 }}\right)$

for $h 2 \quad I_{1}^{D}=\left(I_{3}^{\circ} / 3\right) \eta \lambda_{s} /\left(\eta \lambda_{s}+\lambda_{\text {pol }}\right)$

Resolving the deconvoluted DBARL spectra into 4 components (including the broad component) at $\mathrm{B}=0$, with only $\mathrm{I}_{2}^{\mathrm{D}}$ fixed to $\left(100-4 \mathrm{I}_{3}^{\circ} / 3\right) \%$ gives $\Gamma_{2}=(2.75 \pm 0.02) \mathrm{keV}$ and $\mathrm{I}_{1}^{\mathrm{D}}=(4.9 \pm 0.9) \%$, which compares well with $I_{1}^{D}=4.8 \%$ as expected from $h 1$, eq.(4), by contrast with $I_{1}^{D}=2.9 \%$ as predicted by h2, eq.(5).

4. Conclusion. The present results show that some information can be obtained on the pick-off lifetime of p-Ps by studying the effects of $\mathrm{B}$ in solids using both LS and DBARL or AC. In the present case however, the information is not clear-cut, because of the rather low value of the p-Ps intensity which brings the usefulness and accuracy of the DBARL technique at their extreme limits. Taking this into account, the data indicate that the p-Ps and o-Ps pick-off decay constants are similar. On this basis, it remains that two distinct contact density parameters are necessary to recover the variation with $B$ of both $R$ and $\tau_{4}$. The exact physical meaning of these parameters, and particularly the ascription of ' $\eta^{\prime}$ to the presence of a Ps excited state, is still to be established.

\section{References.}

(1) Billard, I.; Abbé, J. Ch.; Duplâtre, G., Chem.Phys. 127(1988)273.

(2) Halpern, O., Phys.Rev. 94(1954)904.

(3) Mills, A. P. Jr., J.Chem.Phys. 62(1975)2647.

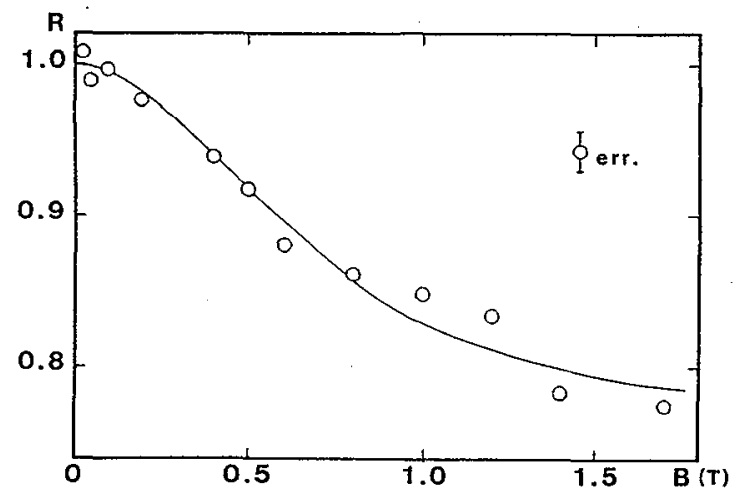

Fig.1. Variation of parameter $\mathrm{R}$ with magnetic field intensity, $B(T)$, in thorium phosphate. Time window : $\mathrm{t}_{\mathrm{a}}=1.8 \mathrm{~ns}, \mathrm{t}_{\mathrm{b}}=$ 5 ns. The solid line is calculated using the parameters from table 1 (treatment d). 